\title{
Estudio micológico del Parque Nacional de Garajonay (La Gomera, Islas Canarias). Agaricales s.l. I
}

\author{
por \\ Ángel Bañares Baudet \& Esperanza Beltrán Tejera \\ Departamento de Biología Vegetal (Botánica), Universidad de La Laguna, E-38071 La Laguna, España \\ angelb@idecnet.com; ebeltran@ull.es
}

\section{Resumen}

Bañares, Á. \& Beltrán, E. 2009. Estudio micológico del Parque Nacional de Garajonay (La Gomera, Islas Canarias). Agaricales s.I. I. Anales Jard. Bot. Madrid 66S1: 47-61.

Tras un reciente estudio de la biota fúngica del Parque Nacional de Garajonay, damos a conocer el catálogo actualizado de Agaricales s.l. de esta área protegida y se describen e ilustran 11 táxones raros o poco conocidos para las Islas Canarias.

Palabras clave: Basidiomycota, Agaricales, corología, taxonomía, Islas Canarias.

\section{Introducción}

Declarado en 1981, el Parque Nacional de Garajonay está situado en la parte central de la isla de La Gomera (Islas Canarias). Sus 3986 hectáreas representan una meseta de complicada orografía, cuyos valles, barrancos y escarpes encuentran su límite superior en la cumbre insular, a los $1400 \mathrm{~m}$ (Alto de Garajonay), llegando a descender por la vertiente norte hasta los $700 \mathrm{~m}$. Alberga las mejores manifestaciones de monteverde (laurisilva y fayal-brezal) cuyo carácter relictual de paleoflora terciaria y excelente grado de conservación dieron pie a que en 1987 fuera reconocido Patrimonio Mundial por la UNESCO.

Sobre un sustrato geológico muy diverso, fundamentalmente basáltico, de aglomerados volcánicos y fonolitas (Bravo \& Bravo, 1990), sus suelos son de tipo ferralítico, pardo ándicos y andisoles, caracterizados por su gran profundidad, reacción ácida y alto contenido en materia orgánica (Jiménez \& al., 1990).

Las cumbres de La Gomera se encuentran en plena influencia del régimen de los vientos alisios del NE, procedentes del anticiclón de las Azores, que al alcan-

\begin{abstract}
Bañares, Á. \& Beltrán, E. 2009. Mycological study Garajonay National Park (La Gomera, Canary Islands). Agaricales s.l. I. Anales Jard. Bot. Madrid 66S1: 47-61 (in Spanish).

After a recent study of the fungi of the Garajonay National Park, an updated catalogue of Agaricales s.I. for this protected area is presented. Furthermore, 11 rare or poorly known species in the Canary Islands are described and illustrated.
\end{abstract}

Keywords: Basidiomycota, Agaricales, chorology, taxonomy, Canary Islands.

zar cotas superiores se condensan, dando lugar a densos "mares de nubes". Constituye por tanto un territorio de clima eminentemente oceánico, templado y húmedo. Entre los 800 y 1000 m, la precipitación media anual a barlovento es de 400 a $900 \mathrm{~mm}$, y la humedad relativa, del 75-90\%; no obstante, a estas cifras hay que añadirle el valioso incremento hídrico producido por la precipitación de nieblas o precipitación horizontal, valor que en ocasiones supera a la precipitación a cielo descubierto (Santana, 1990). Las temperaturas extremas a lo largo del año en la vertiente norte, oscilan de $9-15^{\circ} \mathrm{C}$ y $14-24{ }^{\circ} \mathrm{C}$, a $730 \mathrm{~m}$, y de $5-13^{\circ} \mathrm{C}$ y $11-26^{\circ} \mathrm{C}$, a $1275 \mathrm{~m}$ (Marzol \& al., 1990).

Dentro de la gran convergencia fisionómica existente en el monteverde, aparecen elementos florísticos y comunidades vegetales diversas en función de los contrastes topográficos, humedad, altura, orientación, profundidad del suelo y pendiente. Las comunidades en las que se efectuaron las recolectas fúngicas se agruparon en las 4 grandes unidades ambientales que definieron Pérez de Paz \& al. (1990): laurisilva s.l., fayal-brezal, matorrales de sustitución y comunidades antrópicas, distribuidas en un total de 8 sub- 
unidades, a las que hacemos referencia al inicio del catálogo. Un trabajo posterior (Pérez de Paz \& al., 2008) contempla básicamente estas mismas unidades con algunas variantes, si bien se ve reforzada la denominación de "monteverde" frente a "laurisilva" como término geobotánico más acorde con este tipo de bosque canario. No obstante, la razón de haber seguido la tipología antigua de la vegetación de Garajonay obedece al hecho de haber iniciado bajo esta óptica los trabajos de campo.

Las comunidades de laurisilva constituyen los bosques más umbrófilos e higrófilos del monteverde, en los cuales encuentran su esplendor las especies arbóreas más nobles. Están representadas por 4 variantes. (I) "laurisilva de fondo de barranco con viñátigo" (Lauro novocanariensis-Perseetum indicae, facies de Persea indica), representada por formaciones densas, a menudo de configuración abovedada, ampliamente representada en el areal septentrional y central del Parque. En el estrato arbóreo, aparte del viñátigo (Persea indica), abunda asimismo el laurel (Laurus novocanariensis). (II) "laurisilva de fondo de barranco con til" (Diplazio caudati-Ocoteetum foetentis), relegada a escasos sectores de la vertiente norte, en especial en la Meseta de Vallehermoso, donde el til (Ocotea foetens) domina el estrato arbóreo y en el sotobosque casi exclusivamente sus brinzales. (III) "laurisilva de ladera y meseta" (Lauro novocanariensis-Perseetum indicae) que constituye el monteverde mesófilo donde dominan elementos poco exigentes, como el mencionado laurel, la faya (Myrica faya), el aceviño (Ilex canariensis) y el brezo (Erica arborea). (IV) "laurisilva termófila" (Visneo mocanerae-Arbutetum canariensis), donde encuentran su representación los elementos arbóreos menos exigentes de suelo y humedad, como el mocán (Visnea mocanera), el barbusano (Apollonias barbujana) y el palo blanco (Picconia excelsa), propios asimismo de ambientes de topografía accidentada.

Las comunidades de fayal-brezal abarcan tanto formaciones climácicas como series degradadas del monteverde. Están representadas por las siguientes variantes: (V) "fayal-brezal arboreo" (Violo rivinianae-Myricetum fayae) donde dominan las especies más resistentes, faya, brezo y aceviño, de amplia distribución en mesetas y cotas superiores de la vertiente sur del Parque. (VI, VII) "brezales de crestería", relegados a las crestas cacuminales de la isla, en ambientes venteados, de suelos ácidos y poco profundos, en las que predomina el brezo y abundantes briófitos, líquenes y helechos; la presencia de elementos eminentemente higrófilos (Pericallis appendiculata) frente a otros de acusado xerofitismo (Micromeria lepida) delatan la incidencia de nieblas durante todo el año o ausentes en época estival, respectivamente. (VIII) "brezal de crestería con tejo" (Ilici canariensis-Ericetum platycodonis), una formación singular de las crestas hiperhúmedas que juega un papel importante en los filos del Parque Nacional en torno a los $900 \mathrm{~m}$, protagonizado por el tejo (Erica platycodon), que junto a otros elementos propios del fayal-brezal conforman un bosque relativamente bajo y denso en el estrato superior.

Las afinidades ecológicas de los Agaricales s.l. en cuanto a sustratos y asentamiento a lo largo de los mencionados tipos de vegetación han sido dadas a conocer en un extenso estudio de los hongos del Parque Nacional de Garajonay (Beltrán \& al., 2008), realizado entre 2000 y 2003. El catálogo que presentamos incluye la totalidad de Agaricales s.l. citados para el Parque, habiendo sido identificados la mayor parte de los mismos con motivo del mencionado estudio, cuyos antecedentes se encuentran en los trabajos de Bañares \& Beltrán (1982, 1987, 1990, 1993), Bañares \& al. (1980, 1991), Beltrán \& Bañares (1983), Beltrán \& Wildpret (1975), Cool (1924), Höiland (1979), Rodríguez-Armas \& al. (1983) y Wölfel \& Noordeloos (2001).

\section{Material y métodos}

En el presente trabajo seguimos el concepto tradicional del orden Agaricales, razón por la cual usamos la expresión Agaricales s.l. para referirnos no sólo a los táxones actualmente incluidos en este orden en el sentido estricto, sino también otros que tras las actuales remodelaciones sistemáticas forman parte de los órdenes Boletales, Polyporales y Russulales.

Para la identificación macro y microscópica del material fúngico se empleó una lupa binocular (10-80×) y un microscopio de contraste de fases (Olympus BX 41), al cual se incorporó una cámara digital Olympus Camedia C-5050 para la realización de fotografías. Los caracteres de diagnóstico macro y microscópicos fueron obtenidos a partir de material fresco o bien procedente de herbario, en cuyo caso se realizó rehidratación al 3\% de $\mathrm{KOH}$. Los caracteres biométricos reseñados de las esporas corresponden a mediciones efectuadas de al menos 20. Las autorías de la mayor parte de los táxones corresponden a las reseñadas en la publicación de Authors of Fungal Names (http://www.indexfungorum.org). Todo el material ha sido depositado en el herbario La Laguna, España, sección Micología (TFC Mic).

\section{Táxones estudiados}

Ecología. Te: Terrícola; Li: Lignícola; Co: Corticíco- 
la; Fo: Foliícola; Ca: Carbonícola; Mu: Muscícola; Cop: Coprófila.

Vegetación. Laurisilva s.l.: I, Laurisilva de fondo de barranco con viñátigo; II, Laurisilva de fondo de barranco con til; III, Laurisilva de ladera y meseta; IV, Laurisilva termófila. Fayal-Brezal s.l.: V, Fayal-brezal; VI, Brezal de crestería húmedo (seco en verano); VII, Brezal de crestería hiperhúmedo (húmedo en verano); VIII, Brezal de crestería con tejo. IX, Matorrales de sustitución. X, Comunidades antrópicas.

Agaricus augustus Fr.: Te. V. 1-2-1982 (TFC Mic. 2017).

Agaricus impudicus (Rea) Pilát: Te. I, III. 13-1-1990 (TFC Mic. 6447); 28-11-2000 (TFC Mic. 10770).

Agaricus moelleri Wasser: Te. I, II, III, V. 1-2-1982 (TFC Mic. 2026); 10-11-2000 (TFC Mic. 9757); 11-11-2000 (TFC Mic. 10748).

Agaricus phaeolepidotus (F.H. Møller) F.H. Møller: Te. I, V, VIII. 28-11-2000 (TFC Mic. 10779); 31-10-2001 (TFC Mic. 10714); 13-12-2001 (TFC Mic. 10837).

Agaricus sylvaticus Schaeff.: Te. III. 1-12-1984 (TFC Mic. 2458). Agaricus silvicola (Vittad.) Peck: Te. I. 1-2-1982 (TFC Mic. 2019). Agrocybe aegerita (V. Brig.) Singer: Te. I. 1-1-1981 (TFC Mic. 1989).

Amanita junquillea Quél.: Te. IX, X (Pinus radiata). 12-1-1990 (TFC Mic. 6423).

Amanita muscaria (L.) Lam: Te (Cistus monspeliensis). X. Amanita pantherina (DC.) Krombholz: Te (Cistus monspeliensis). V, VII. 10-12-2000 (TFC Mic. 10804).

Amanita rubescens Pers.: Te. V, X (Castanea sativa).

Armillaria mellea (Vahl) P. Kumm.: Li, Co (Persea indica y Laurus novocanariensis). III. 1-12-1983 (TFC Mic. 2426); 11-11-2000 (TFC Mic. 9761).

Boletus edulis Bull.: Te. X (Pinus radiata). 2-1-1977 (TFC Mic. 1227).

Calocybe onychina (Fr.) Donk: Li (Erica arborea). X. 30-112002 (TFC Mic. 10848).

Cheimonophyllum candidissimum (Berk. \& M.A. Curtis) Singer (Fig. 1): Li, Co (Myrica faya, Laurus novocanariensis, Persea indica y Eucaliptus globulus). I, II, III, VIII, X. 2-1-1977 (TFC Mic. 811); 1-12-1983 (TFC Mic. 2414); 12-2-2000 (TFC Mic. 10761); 13-22000 (TFC Mic. 10762); 11-11-2000 (TFC Mic. 9764); 28-11-2000 (TFC Mic. 10824); 29-11-2000 (TFC Mic. 10819); 16-5-2001 (TFC Mic. 10726); 29-10-2001 (TFC Mic. 10712, 10713).

Chlorophyllum rhacodes (Vittad.) Vellinga: Te. II. 1-2-1982 (TFC Mic. 2016).

Clitocybe anisata Velen.: Te. X (Eucaliptus globulus). 1-11-1989 (TFC Mic. 6433).

Clitocybe costata Kühner \& Romagn.: Te. X (Pinus radiata). 1611-1987 (TFC Mic. 3981); 12-12-1987 (TFC Mic. 3983).

Clitocybe metachroa (Fr.) P. Kumm.: Te. V, X (Pinus radiata). 12-12-1987 (TFC Mic. 3975); 10-12-2000 (TFC Mic. 9922); 21-12001 (TFC Mic. 9958).

Clitocybe pruinosa (Lasch) P. Kumm.: Te. V, X. 10-12-2000 (TFC Mic. 10805).

Clitocybe rivulosa (Pers.) P. Kumm.: Te. I, III, V, X (Ficus carica). 1-2-1982 (TFC Mic. 2003); 11-11-2000 (TFC Mic. 10751); 201-2001 (TFC Mic. 10722).

Clitocybe trulliformis (Fr.) P. Karst.: Te. X (Ficus carica, Pinus radiata). 1-12-1984 (TFC Mic. 2452); 19-1-2001 (TFC Mic. 9945); 20-1-2001 (TFC Mic. 10719).

Clitocybula wildpretii (Bañares, Beltrán-Tej. \& Bon) Esteve-
Rav., Barrasa \& Bañares: Li. V, VII. 20-1-2001 (TFC Mic. 9953); 13-12-2001 (TFC Mic. 10838).

Coprinellus micaceus (Bull.) Vilgalys, Hopple \& Jacq. Johnson: $\mathrm{Te}, \mathrm{Li}$ (Laurus novocanariensis). I, III, V, VII, VIII. 12-2-2000 (TFC Mic. 10760); 29-11-2000 (TFC Mic. 10775); 16-5-2001 (TFC Mic. 10730)

Contumyces rosellus (M.M. Moser) Redhead, Moncalvo, Vilgalys \& Lutzoni: Te (calveros). VII. 11-1-1990 (TFC Mic. 6417).

Cortinarius croceus (Schaeff.) Gray: Te. X (Pinus radiata). 12-11990 (TFC Mic. 6446).

Crepidotus calolepis (Fr.) P. Karst. var. calolepis: Li. II, III. 21982 (TFC Mic. 1998); 2-12-2001 (TFC Mic. 10833).

Crepidotus mollis (Schaeff.) Staude: Li. I, III, VI. 25-2-1973 (TFC Mic. 282).

Crinipellis phyllophila Singer: Fo (Myrica faya). III, V, VIII, X. 1-2-1982 (TFC Mic. 2021); 12-11-2000 (TFC Mic. 9794); 29-112000 (TFC Mic. 10817); 2-12-2001 (TFC Mic. 10831).

Cystodermella cinnabarina (Alb. \& Schwein.) Harmaja var. cinnabarina: Te. X (Eucaliptus globulus). 11-1-1990 (TFC Mic. 6448).

Entoloma serrulata (Pers.) Hesler: Te. III, V, VII, VIII. 13-11990 (TFC Mic. 6453); 28-11-2000 (TFC Mic. 10767); 21-1-2001 (TFC Mic. 10721).

Faeberia carbonaria (Alb. \& Schwein.) Pouzar: Ca. V. 6-121987 (TFC Mic. 3978).

Flammulaster carpophilus (Fr.) Earle: Te. IX (Cistus monspeliensis), X (Eucaliptus globulus). 11-1-1990 (TFC Mic. 6443).

Flammulina velutipes (Curtis) Singer: Te. III. 1-10-1987 (TFC Mic. 3973).

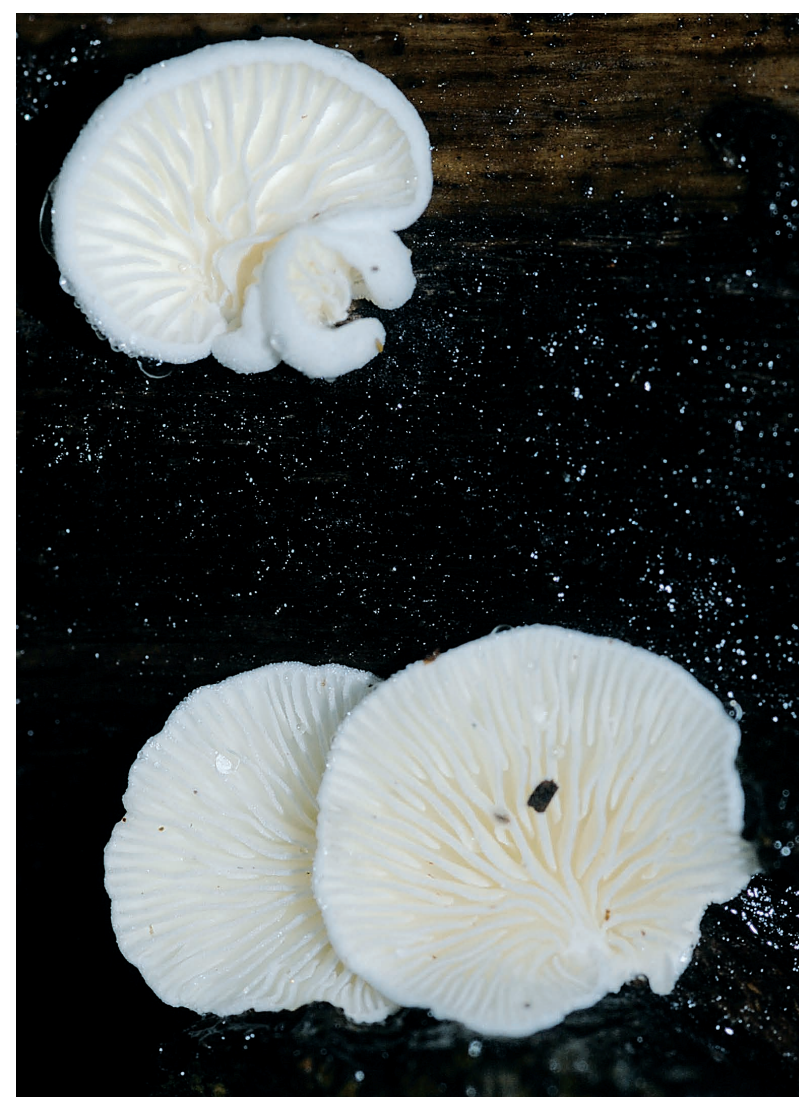

Fig. 1. Cheimonophyllum candidissimum. Basidiomas, Bañares 10761 (TFC Mic.). 

III.

Galerina bypnorum (Schrank) Kühner: Mu (Polytrichum sp.).

Galerina unicolor (Vahl) Singer: Li. V, IX, X (Pinus radiata). 11-1982 (TFC Mic. 2031); 10-12-2000 (TFC Mic. 9921); 30-112002 (TFC Mic. 10850).

Gymnopilus junonius (Fr.) P.D. Orton: Li, Co (Eucaliptus globulus, Ilex canariensis). I, II, X. 2-1-1977 (TFC Mic. 768).

Gymnopilus sapineus (Fr.) Maire: Li (Myrica faya, Erica arborea, Pinus canariensis, P. radiata). III, V, IX, X. 1-1-1982 (TFC Mic. 2030); 10-12-2000 (TFC Mic. 10802).

Gymnopus beltraniae Bañares, Antonín \& G. Moreno (Fig. 2): Te, Li. I, III, V, VII. 29-11-2000 (TFC Mic. 10842); 12-11-2000 (TFC Mic. 9703); 11-11-2000 (TFC Mic. 9768); 11-11-2000 (TFC Mic. 9773); 21-1-2001 (TFC Mic. 10843); 20-1-2001 (TFC Mic. 10844).

Gymnopus dryophilus (Bull.) Murril: Te. III. 1-1-1981 (TFC Mic. 1988)

Hebeloma cistophilum Maire: Te (Cistus monspeliensis). III, V. 12-11-2000 (TFC Mic. 9786; 9800); 13-12-2001 (TFC Mic. 10835).

Hebeloma crustuliniformis (Bull.) Quél.: Te. X (Pinus radiata, Castanea sativa). 12-1-1990 (TFC Mic. 6429).

Hohenbuehelia mastrucata (Fr.) Singer: Li (Ilex canariensis). V. 1-10-1987 (TFC Mic. 3972).

Hygrocybe cantharellus (Schwein.) Murrill: Te. I, II, III. 1-2 1981 (TFC Mic. 1861); 13-2-2000 (TFC Mic. 10765).

Hygrocybe chlorophana (Fr.) Wünsche: Te. III. 28-11-2000 (TFC Mic. 10827); 1-5-2001 (TFC Mic. 10809).

Hygrocybe conica (Schaeff.) P. Kumm.: Te. I, II, II, V, X. 2-11977 (TFC Mic. 782); 28-11-2000 (TFC Mic. 10771); 29-11-2000

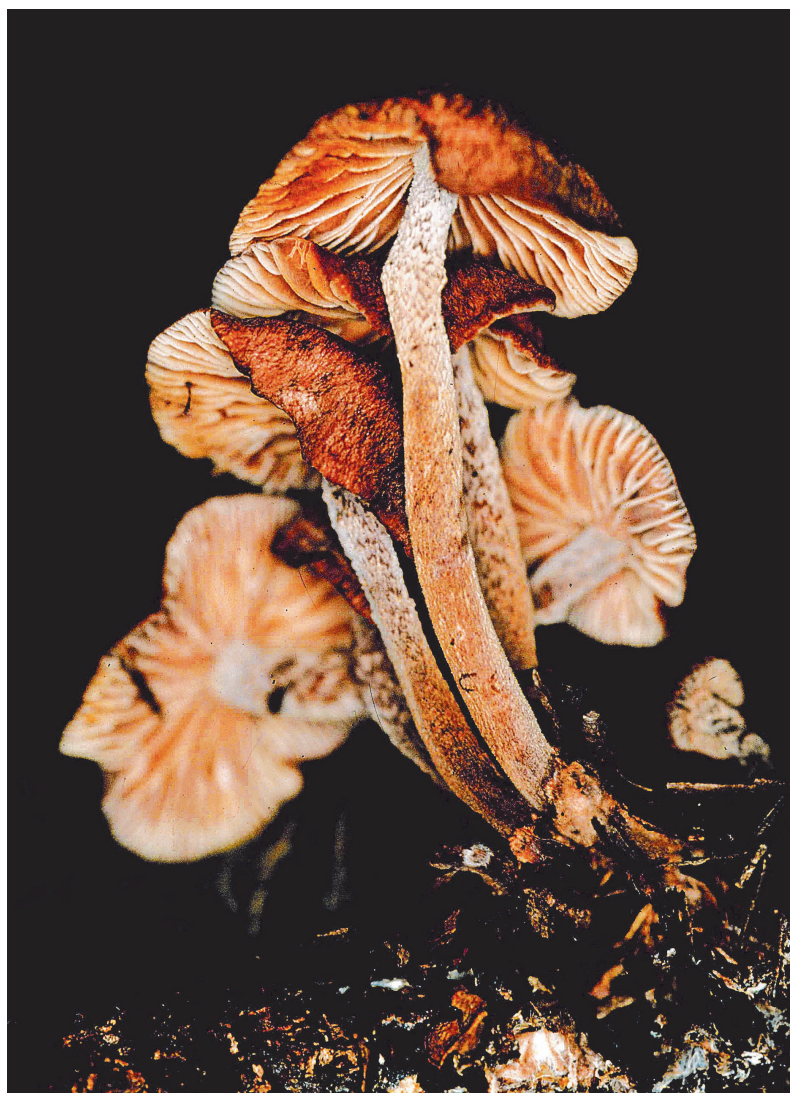

Fig. 2. Gymnopus beltraniae. Basidiomas, Bañares 10844 (TFC Mic.).
(TFC Mic. 10818); 12-11-2000 (TFC Mic. 9805); 21-1-2001 (TFC Mic. 10746).

Hygrocybe glutinipes (J.E. Lange) R. Haller Aar. var. glutinipes:

Te. VII. 20-1-2001 (TFC Mic. 10737).

Hygrocybe miniata (Fr.) P. Kumm.: Te, Mu. I. 13-2-2000 (TFC

Mic. 10750); 29-11-2000 (TFC Mic. 10786).

Hygrocybe mucronella (Fr.) P. Karst.: Te. III. 28-11-2000 (TFC Mic. 10822).

Hygrocybe pratensis (Pers.) Murril: Te. III, X (Castanea sativa).

2-1-1977 (TFC Mic. 1228); 28-11-2000 (TFC Mic. 10706).

Hygrocybe psittacina (Schaeff.) P. Kumm.: Te. III. 12-11-2000

(TFC Mic. 10695).

Hygrocybe punicea (Fr.) P. Kumm.: Te. III. 1-1-1981 (TFC Mic. 1885); 28-11-2000 (TFC Mic. 10777)

Hygrocybe reidii Kühner: Mu. I. 29-11-2000 (TFC Mic. 10780). Hygrocybe russocoriacea (Berk. \& Jos. K. Mill.) P.D. Orton \& Watling: Te. I. 1-1-1984 (TFC Mic. 2412); 28-11-2000 (TFC Mic. 10826); 20-1-2001 (TFC Mic. 10747). 284).

Hygrocybe turunda (Fr.) P. Karst.: Te. III. 25-2-1973 (TFC Mic.

Hygrocybe virginea (Wulfen) P.D. Orton \& Watling: Te. III. 16-5-2001 (TFC Mic. 10729).

Hygrophoropsis aurantiaca (Wulfen) Maire: Mu. III. 1-12-83

(TFC Mic. 2421)

Inocybe acuta Boud.: Te. III.

Laccaria laccata (Scop.) Cooke: Te. X (Pinus radiata). 11-1-1990

(TFC Mic. 6411)

Laccaria fraterna (Cooke \& Massee) Pegler.: Te. X (Eucaliptus globulus). 1-1-1981 (TFC Mic. 1930).

Laccaria proxima (Boud.) Pat.: Te. X (Pinus radiata). 1-1-1981 (TFC Mic. 1855).

Lacrymaria lacrimabunda (Bull.) Pat.: Te. I. 1-1-1981 (TFC Mic. 1905).

Lactarius cistophilus Bon \& Trimbach: Te (Cistus monspeliensis). III.

Lactarius deliciosus (L.) Gray: Te. X (Castanea sativa).

Lactarius tesquorum Malençon: Te (Cistus monspeliensis). X (Pinus radiata).

Leccinum corsicum (Rolland) Singer: Te (Cistus monspeliensis). IX. 19-1-2001 (TFC 10789).

Lentinellus flabelliformis (Bolton) S. Ito: Li. V, X. 29-11-2000 (TFC Mic. 10823); 30-11-2002 (TFC Mic. 10847, 10846).

Lentinellus micheneri (Berk. \& M.A. Curtis) Pegler: Li (Chamaecytisus proliferus, Pinus radiata, Cistus monspeliensis). IX, X. 1-11981 (TFC Mic. 1986); 10-12-2000 (TFC Mic. 10803).

Lepiota clypeolaria (Bull.) P. Kumm.: Te. III, X (Pinus radiata). 1-11-1984 (TFC Mic. 2413); 1-11-1989 (TFC Mic. 6425); 1-111989 (TFC Mic. 6427).

Lepiota pseudolilacea Huijsman: Te. III. 13-12-2001 (TFC Mic. 10834).

Lepista nuda (Bull.) Cooke: Te. III, X (Eucaliptus globulus). 11-1982 (TFC Mic. 2015); 29-11-2000 (TFC Mic. 10702).

Lepista ovispora (J.E. Lange) Gulden: Te. III, V. 29-11-2000 (TFC Mic. 10759); 13-12-2001 (TFC Mic. 10839).

Leucoagaricus crystallifer Vellinga (Fig. 3): Te. III. 15-11-1983 (TFC Mic. 2404); 12-11-2000 (TFC Mic. 10752); 13-12-2001 (TFC Mic. 10836).

Leucoagaricus melanotrichus (Malençon \& Bertault) Trimbach: Te. V, IX. 10-11-2000 (TFC Mic. 10694); 19-1-2001 (TFC Mic. 9942).

Leucoagaricus purpureolilacinus Huijsman (Fig. 4): Te. V. 1-101987 (TFC Mic. 3400)

Leucopaxillus gentianeus (Quél.) Kotl.: Te. III, V, VI, VII, X (Castanea sativa). 1-11-1989 (TFC Mic. 6441); 10-11-2000 (TFC Mic. 9753); 12-11-2000 (TFC Mic. 10698). 
Lyophyllum decastes (Fr.) Singer: Te. X (Pinus radiata).

Macrocystidia cucumis (Pers.) Joss. f. cucumis: Te. III, V. 28-112000 (TFC Mic. 10769); 29-11-2000 (TFC Mic. 10776).

Marasmius corbariensis (Roumeg.) Singer: Fo (Picconia excelsa). III. 28-4-2001 (TFC Mic. 10806).

Marasmius epiphylloides var. latispora Bañares \& Beltrán: Fo (Hedera helix ssp. canariensis). V. 11-12-1990 (TFC Mic. 6372).

Marasmius budsonii (Pers.) Fr.: Fo (Ilex canariensis, I. perado ssp. platyphylla). I, II, III, V, VI, X. 1-1-1982 (TFC Mic. 2024); 13 2-2000 (TFC Mic. 10700); 18-5-2000 (TFC Mic. 10754); 10-112000 (TFC Mic. 10697); 28-11-2000 (TFC Mic. 10705); 10-122000 (TFC Mic. 10814).

Marasmius scorodonius (Fr.) Fr. var. scorodonius: Te, Li. III, VII. 1-11-1983 (TFC Mic. 2425)

Marasmius scorodonius var. virgultorum Malençon \& Bertault: Te, Li (Cistus monspeliensis). V. 10-12-2000 (TFC Mic. 10800, Dup. in BRNM); 30-10-2001 (TFC Mic. 10715).

Megacollybia platyphylla (Pers.) Kotl. \& Pouzar: Li. II, V, VIII. 1-2-1982 (TFC Mic. 1997); 28-11-2000 (TFC Mic. 10701); 30-102001 (TFC Mic. 10709).

Mycena acicula (Schaeff.) P. Kumm.: Li (Erica arborea). X. 2911-2000 (TFC Mic. 10821).

Mycena adonis (Bull.) Gray: Li. III. 12-11-2000 (TFC Mic. 9806); 28-4-2001 (TFC Mic. 10693).

Mycena adscendens (Lasch) Maas Geest. (Fig. 5): Li. I, II, III. 13-1-1990 (TFC Mic. 4412); 10-1-1990 (TFC Mic. 6431); 11-11990 (TFC Mic. 6438); 13-2-2000 (TFC Mic.10756).

Mycena amicta (Fr.) Quél.: Te. X (Pinus radiata).

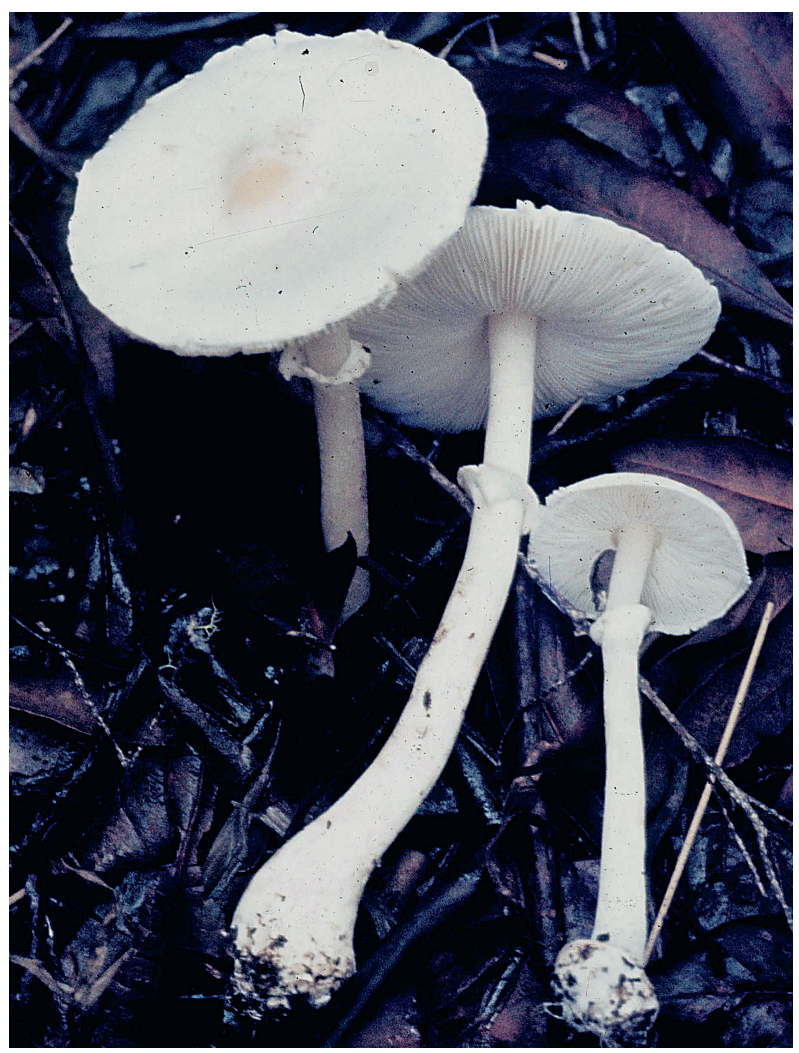

Fig. 3. Leucoagaricus crystallifer. Basidiomas, Bañares 10836 (TFC Mic.).

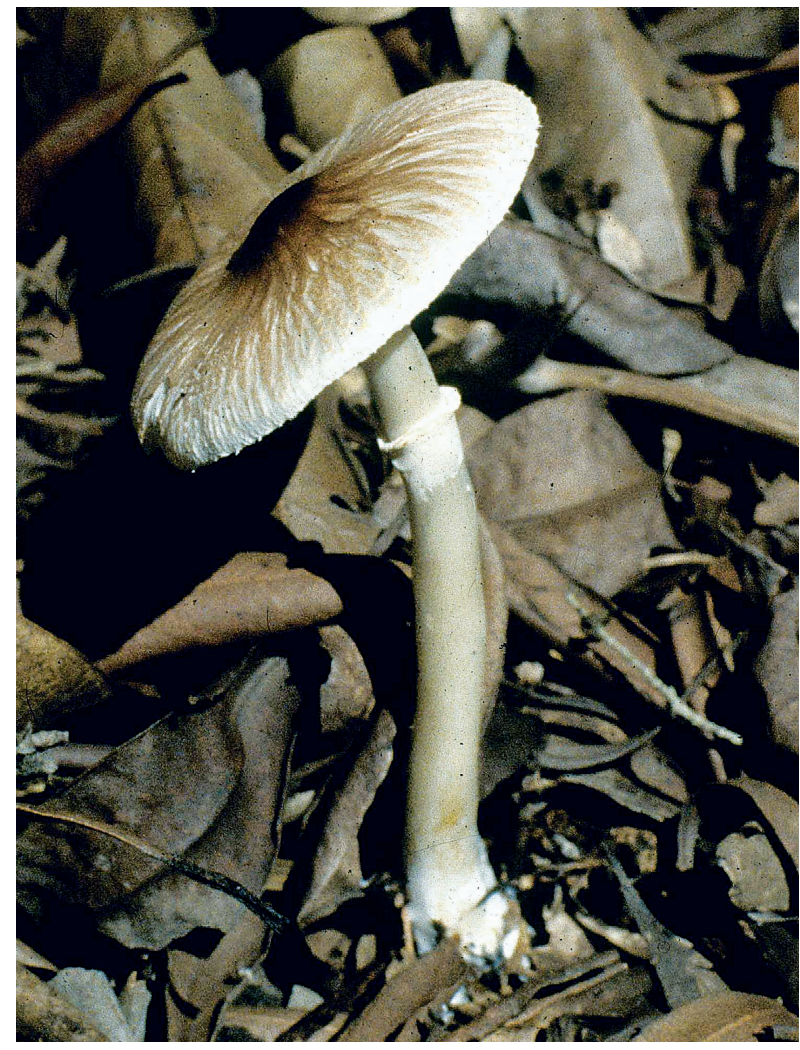

Fig. 4. Leucoagaricus purpureolilacinus. Basidioma, Bañares 3400 (TFC Mic.).

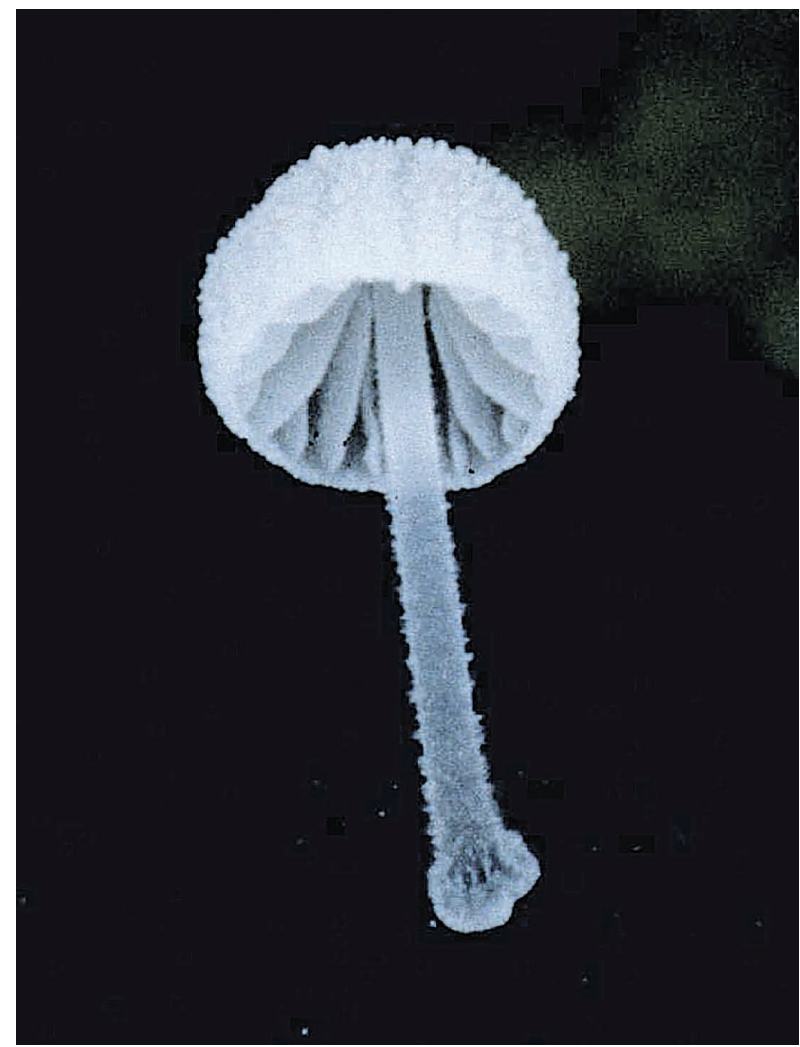

Fig. 5. Mycena adscendens. Basidioma, Bañares 10756 (TFC Mic.). 
Mycena epipterygia (Scop.) Gray var. epipterygia: Li (Erica arborea). III. 12-11-2000 (TFC Mic. 9821).

Mycena galericulata (Scop.) Gray: Li. I, II, III, V, VII, X. 6-121987 (TFC Mic. 3980); 28-11-2000 (TFC Mic. 10783); 29-11-2000 (TFC Mic. 10773); 29-11-2000 (TFC Mic. 10774); 12-11-2000 (TFC Mic. 9819).

Mycena haematopus (Pers.) P. Kumm.: Li (Laurus novocanariensis). I. 30-11-2002 (TFC Mic. 10849).

Mycena inclinata (Fr.) Quél.: Li. III, V, X. 2-1-1977 (TFC Mic. 812); 28-11-2000 (TFC Mic. 10793); 29-11-2000 (TFC Mic. 10785); 12-11-2000 (TFC Mic. 9810).

Mycena mirata (Peck) Sacc.: Fo (Myrica faya). V. 20-1-2001 (TFC Mic. 10724)

Mycena pura (Pers.) P. Kumm.: Te. III, V, VII, X. 1-1-1981 (TFC Mic. 1904); 12-11-2000 (TFC Mic. 10699); 20-1-2001 (TFC Mic. 10696).

Mycena renatii Quél.: Li (Laurus novocanariensis). III. 29-102001 (TFC Mic. 10710).

Mycena sanguinolenta (Alb. \& Schwein.) P. Kumm. var. sanguinolenta: Li, Mu. III, V, VI. 20-1-2001 (TFC Mic. 10717); 28-42001 (TFC Mic. 10808); 30-11-2002 (TFC Mic. 10.854).

Mycena stylobates (Pers.) P. Kumm.: Fo (Myrica faya), Li. V, X. 10-12-2000 (TFC Mic. 10815).

Mycena urania (Fr.) Quél.: Te. VII. 10-1-1990 (TFC Mic. 6422).

Omphalotus olearius (DC.) Singer: Li. III. 11-1-1990 (TFC Mic. 6444).

Panaeolus papilionaceus (Bull.) Quél.: Cop. (asno). V. 1-121984 (TFC Mic. 2447).

Panellus stypticus (Bull.) P. Karst.: Li. I, III, X (Pinus radiata). 11-1981 (TFC Mic. 1847); 13-2-2000 (TFC Mic. 10753); 15-4-2000 (TFC Mic. 10764); 10-12-2000 (TFC Mic. 9926); 1-5-2001 (TFC Mic. 10692, 10691).

Phaeomarasmius erinaceus (Pers.) Scherff. ex Romagn.: Li (Erica arborea). V. 12-11-2000 (TFC Mic. 9801).

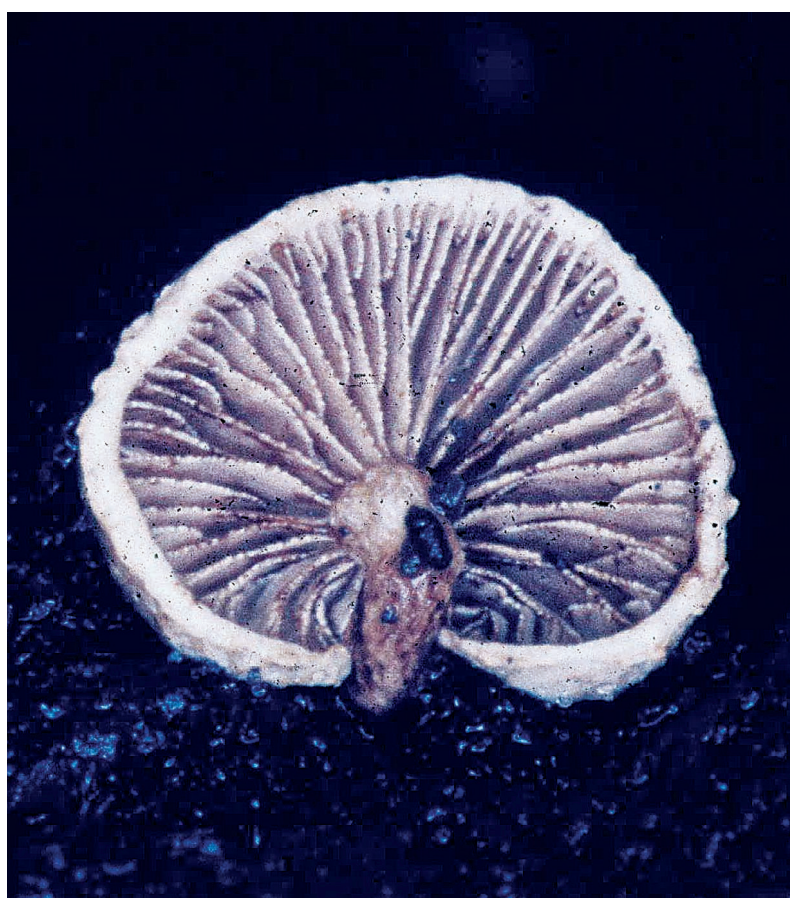

Fig. 6. Pleuroflammula raggaziana. Basidioma, Bañares 10716 (TFC Mic.).
Pholiota highlandensis (Peck) A.H. Sm. \& Hesler: Ca. X (Pinus radiata). 18-10-1987 (TFC Mic. 3974).

Pleuroflammula raggaziana (Bres.) E. Horak (Fig. 6): Li. I, II, III. 11-11-2000 (TFC Mic. 9779); 1-5-2001 (TFC Mic. 10810); 2910-2001 (TFC Mic. 10716); 18-7-2002 (TFC Mic. 10840).

Pleurotus dryinus (Pers.) P. Kumm.: Li (Ilex canariensis). I. 112-1981 (TFC Mic. 2027); 1-2-1982 (TFC Mic. 2008).

Pluteus cervinus (Schaeff.) P. Kumm.: Li. I, III, X (Eucaliptus globulus). 1-1-1982 (TFC Mic. 2014).

Pluteus insidiosus Vellinga \& Schreurs: Te. III. 16-5-2001 (TFC Mic. 10791).

Pluteus plautus (Weinm.) Gillet: Li (Eucaliptus globulus). X. 111-1990 (TFC Mic. 6410).

Pluteus salicinus (Pers.) P. Kumm.: Li. I, III, V, VI. 1-1-1982 (TFC Mic. 1994); 1-1-1982 (TFC Mic. 2023); 13-2-2000 (TFC Mic. 10703).

Psathyrella candolleana (Fr.) Maire: Te. I, V, X (Castanea sativa).

Psathyrella gracilis (Fr.) Quél.: Te. III, X. 1-11-1989 (TFC Mic. 6426); 29-11-2000 (TFC Mic. 10778).

Pseudoclitocybe cyathiformis (Bull.) Singer: Te, Li. X (Eucaliptus globulus). 1-1-1982 (TFC Mic. 2010).

Psilocybe fascicularis (Huds.) Noordel.: Li. I, III, V. 12-11-2000 (TFC Mic. 9809).

Resupinatus kavini (Pilát) M.M. Moser: Li. III, VIII. 1-5-2001 (TFC Mic. 10799); 1-5-2001 (TFC Mic. 10796); 30-10-2001 (TFC Mic. 10708).

Resupinatus unguicularis (Fr.) Singer (Fig. 7): Li, Co (Laurus novocanariensis, Myrica faya). III, V. 13-10-2001 (TFC Mic. 10707); 2-12-2001 (TFC Mic. 10830); 30-11-2002 (TFC Mic. 10857).

Rhodocollybia butyracea (Bull.) Lennox: Te. I, III, V, VII, X. 11-1981 (TFC Mic. 1879); 29-11-2000 (TFC Mic. 10704); 12-112000 (TFC Mic. 9804).

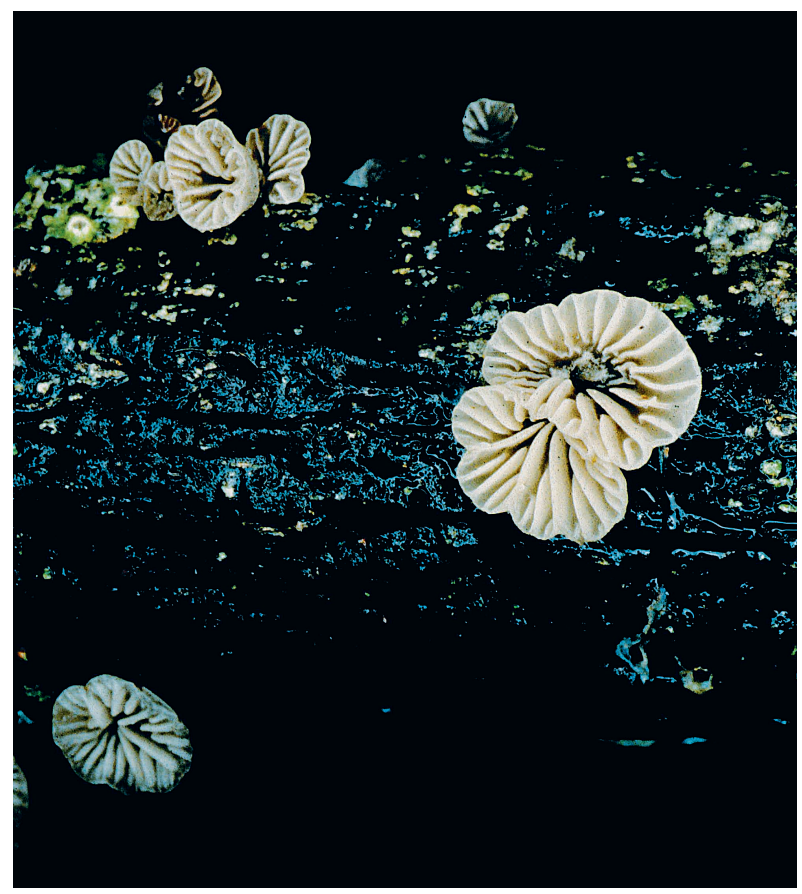

Fig. 7. Resupinatus unguicularis. Basidiomas, Bañares 10830 (TFC Mic.). 
Rickenella fibula (Bull.) Raithelh.: Mu. VII. 29-11-2000 (TFC Mic. 10825).

Roridomyces roridus (Scop.) Rexer: Fo (Myrica faya). V. 21-12001 (TFC Mic. 10733).

Russula albonigra (Krombh.) Fr.: Te. IX. 1-1-1989 (TFC Mic. 6449).

Russula aurea Pers.: Te. X. 1-11-1989 (TFC Mic. 6420).

Russula delica Fr.: Te. X (Castanea sativa, Pinus radiata). 1-111989 (TFC Mic. 6421).

Suillus bellinii (Inzenga) Watling: Te. X (Pinus radiata).

Tapinella panuoides (Batsch) E.-J. Gilbert: Li. X (Pinus radiata). 12-1-1990 (TFC Mic. 6436).

Tricholoma focale (Fr.) Ricken: Te. X (Pinus radiata). 6-12-1987 (TFC Mic. 3979).

Tricholoma saponaceum (Fr.) P. Kumm.: Te. X (Pinus radiata). 1-1-1981 (TFC Mic. 1987).

Tricholomopsis rutilans (Schaeff.) Singer: Li (Pinus canariensis). X. 11-1-1990 (TFC Mic. 6415).

Tubaria conspersa (Pers.) Fayod: Te, Li. III, V. 10-1-1990 (TFC Mic. 6435); 16-5-2001 (TFC Mic. 10790.

Tubaria romagnesiana Arnolds: Li. II. 1-10-1987 (TFC Mic. 3971).

\section{Táxones raros o poco conocidos}

Clitocybe cistophila Bon \& Contu, Doc. Mycol. 60: 43. 1985

Píleo de 1,5-2 cm de diámetro, convexo, después extendido y finalmente algo deprimido en el centro, de un beige claro, liso, higrófano; margen incurvado. Láminas adheridas a subdecurrentes, apretadas, gruesas; lamélulas presentes. Estípite de 22-26 × 3,5-5,5 $\mathrm{mm}$, más o menos cilíndrico o algo atenuado hacia el ápice, blanquecino y algo pruinoso, la base exhibe micelio blanco abundante y algunos rizomorfos. Carne con fuerte olor a anís. Esporas de 4,8-6,6(7,2) × 3,4$4,1(4,4) \mu \mathrm{m}$, elipsoides, acianófilas. Pileipellis de hifas $\times 4-10 \mu \mathrm{m}$, poco ramificadas, a veces diverticuladas. Fíbulas abundantes.

Observaciones. Nuestro material coincide bien con la descripción original de este taxon ligado a especies del género Cistus, del subgénero Pseudolyophyllum Singer emend. ss. lato, sección Fragantes Harm. ex Bon; no obstante, las esporas son algo mayores.

C. anisata Velen., también presente en la zona de estudio, se diferencia por su mayor porte (píleo de 3-5 cm de diámetro), láminas más espaciadas, esporada con tonalidades rosadas y su hábitat no ligado a Cistus.

Ecología. Te. IX (Cistus monspeliensis). 2-12-2001 (TFC Mic. 10829).

Clitocybe nivea Velen., Ceske Houby: 255. 1920 (Fig. 8)

$=$ C. bypotheja Bellú
Carpoforos a menudo cespitosos. Píleo de 3,5-7 cm de diámetro, convexo a extendido, generalmente algo umbonado, carnoso, de un blanco mate y opaco, seco; margen generalmente incurvado. Láminas decurrentes a fuertemente decurrentes, más o menos espaciadas, de un blanco-crema a ocráceas que en exsiccata oscurecen a beige. Estípite de 4,5-7 × 0,6-1 cm, concolor al píleo y base algo ensanchada. Carne de sabor dulzaino. Esporas de 5,9-7,7 × 3,6-4,4(4,8) $\mu \mathrm{m}$, oblongas a subamigdaliformes, con apícula más o menos pronunciada, dispuestas tanto en tétradas como aisladas. Esporada crema-ocrácea. Cistidios ausentes. Basidios grandes, de 26-40 × 5,6-6,8 $\mu \mathrm{m}$, más o menos cilíndricos a subclaviformes, con esterigmas largos, tetraspóricos pero también 1 y 2 espóricos. Pileipellis de hifas anchas $(\times 6,4-7 \mu \mathrm{m})$ con pigmento parietal, a menudo provistas de protuberancias, alternando con hifas estrechas $(\times 2,5-3,8 \mu \mathrm{m})$, sinuosas y asimismo diverticuladas, de ápice redondeado. Fíbulas muy patentes.

Observaciones. Nuestro material coincide plenamente con la descripción de Bon (1997) y Migliozzi \& Cambonii (1999) de este taxon del subg. Hygroclitocybe Bon, secc. Geotropa Bon, caracterizado a grandes rasgos por su coloración de un blanco intenso, láminas espaciadas y fuertemente decurrentes (que recuerdan a Hygrophorus), así como por sus esporas grandes y basidios muy elongados.

En el área mediterránea ha sido recientemente citada por Bellú (1996), Consiglio (1997) y Tabarés (1996). A primera vista resulta fácil de confundir con las especies de la sección Candicantes (Quél.) Konrad \& Maubl., en especial con C. phyllophylla (Pers.) P. Kumm. y C. rivulosa (Pers.) P. Kumm.; no obstante, se diferencia claramente por sus láminas fuertemente decurrentes y por sus esporas y basidios mayores. De esta última especie, presente en la zona de estudio, se diferencia asimismo por su carácter gregario y porte más robusto.

Ecología. Te. I, III, VII. 28-11-2000 (TFC Mic. 10772); 28-11-2000 (TFC Mic. 10782); 9-12-2000 (TFC Mic. 10801); 2-12-2001 (TFC Mic. 10832).

Crepidotus calolepis var. squamulosus (Cout.) SennIrlet, Persoonia 16(1): 37. 1995

Siguiendo a Senn-Irlet (1995), se diferencia de la variedad típica, asimismo presente en la zona de estudio, por sus esporas y escamas del píleo mayores. En nuestro material las esporas miden $8,3-10,7(11,8) \times$ 6,3-7,5(8,1) $\mu \mathrm{m}$ (en la variedad típica 7,4-9,3 × 5,2-6,8 $\mu \mathrm{m})$. No obstante, las escamas del píleo aparecen con dimensiones semejantes o algo menores, de 6,3-14,8 $\mu \mathrm{m}$ de ancho (en la variedad típica 6,5-17 $\mu \mathrm{m}$ de an- 
cho). Por otro lado, los carpóforos presentan dimensiones superiores a la var. calolepis, alcanzando $6 \mathrm{~cm}$ de diámetro.

Ecología. Li. III. 16-5-2001 (TFC Mic. 10792).

Crepidotus cesatii (Rabenh.) Sacc., Michelia 1(no. 1): 2. 1877 , var. cesatii

$=$ Crepidotus sphaerosporus (Pat.) J. Lange.; Crepidotus cesatii var. sphaerosporus (Pat.) A. Ortega \& Buendía

Píleo de 5-15 mm de diámetro, sésil, fijado lateralmente al sustrato, al principio ungulado, reniforme, después más o menos circular, con el margen involuto, blanco mate, de superficie afieltrado-tomentosa a subtomentosa. Láminas de un crema asalmonado, después canelas, estrechamente adheridas; lamélulas muy escasas. Esporada marrón-rojizo. Esporas de 6,68,1 × 5,2-7,2 $\mu \mathrm{m}$, subglobosas con apícula notoria, equinuladas. Queilocistidios estrechamente utriformes, fuertemente ramificados en el ápice. Pileipellis de hifas (2,6)3,4-6 um de ancho, a menudo enrolladas. Fíbulas presentes.

Observaciones. Nuestro material coincide bien con la descripción de Senn-Irlet (1995). La morfología esporal subglobosa y la pileipellis de hifas a menudo enrolladas, le diferencia de la var. subsphaerosporus (J.E. Lange) Senn-Irlet. En la zona de estudio C. cesatii puede confundirse a primera vista con Cheimonophyllum candidissimum (Berk. \& M.A. Curtis) Singer, pero éste presenta láminas siempre blancas y esporas esféricas, lisas.

Ecología. Li (Laurus novocanariensis, Adenocarpus foliolosus), Fo (Myrica faya). III, VI, VII, VIII. 12-22000 (TFC Mic. 10763); 12-2-2000 (TFC Mic. 10766); 20-1-2001 (TFC Mic. 10735); 30-11-2002 (TFC Mic. 10856).

Crepidotus lundellii Pilát, Fungi Exsicc. Suecici fasc.V-VI: 10. 1936 (Fig. 9)

= C.amygdalosporus Kühner; C. subtilis P.D. Orton; C. versutus var. subglobisporus Pilát

Píleo de 5-20 mm de diámetro, al principio campanulado, después extendido, redondeado, sésil o provisto de un pequeño estípite lateral de $1-2 \times 0,5$ $1 \mathrm{~mm}$, fijado al dorso, con la base densamente villosotomentosa, de un blanco-crema a algo ocráceo, superficie fibrillo-tomentosa; margen al principio incurvado. Láminas adheridas, blanquecinas después beiges a marrones, de margen fimbriado. Esporas de $(6,1) 7,4-8,8 \times 4,4-5,9 \mu \mathrm{m}$, ovoides a subelipsoides, sublisas (oscuramente verrucosas), débilmente coloreadas. Pileipellis de elementos cilíndricos, de 4,1-8,1 $\mu \mathrm{m}$ de ancho, a menudo ramificados o provistos de cortas digitaciones y provistos de fíbulas. Queilocistidios de 6-14,8 $\mu \mathrm{m}$ de ancho, hialinos, estrechamente utriformes, raramente claviformes, subcilíndricos, fusiformes, con una protuberancia apical gruesa y obtusa, mamiforme, a veces septados.

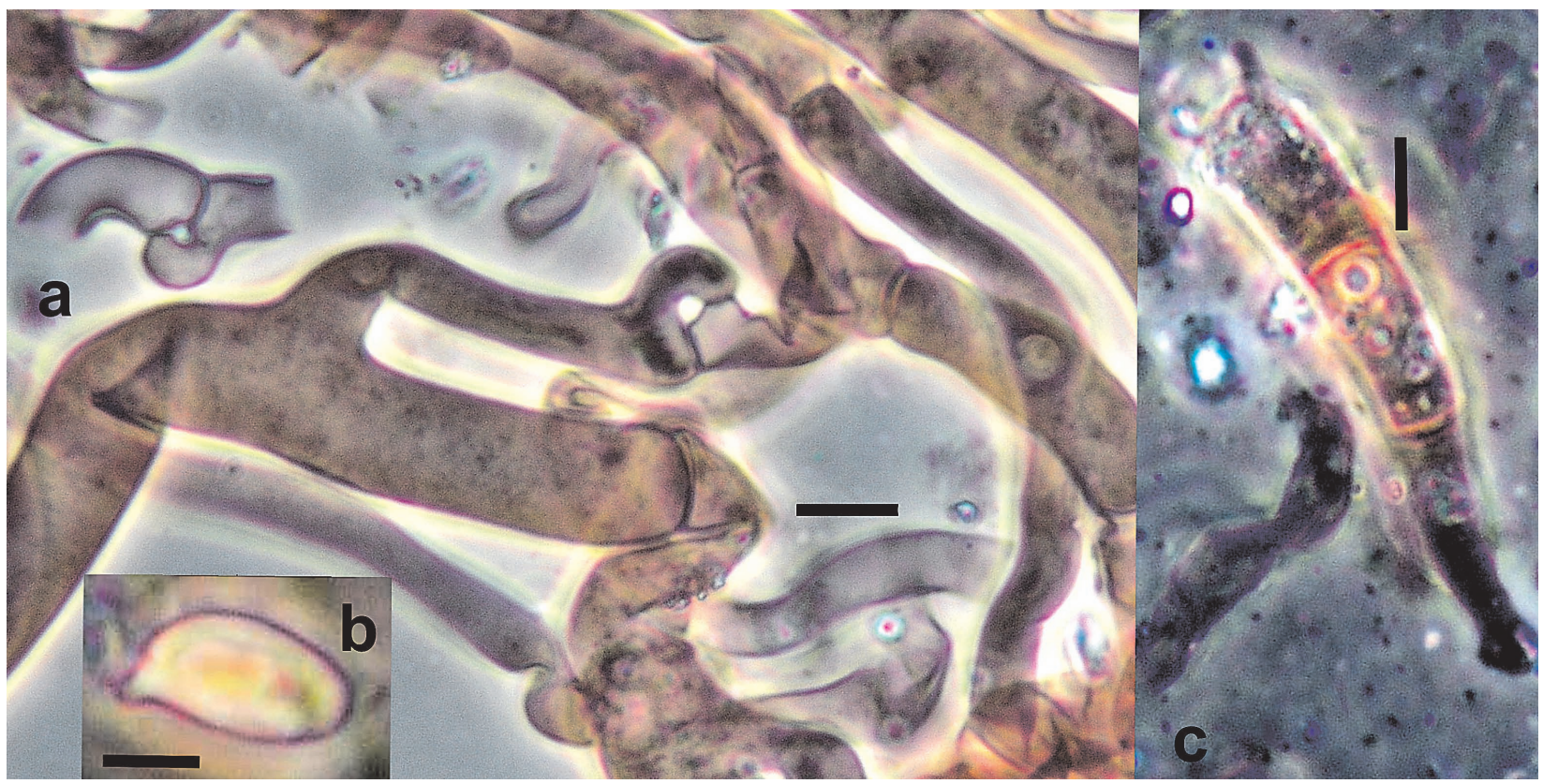

Fig. 8. Clitocybe nivea: a, pileipellis; b, espora; c, basidio. Bañares 10782 (TFC Mic.). Barras: a, c = $5 \mu \mathrm{m} ; \mathrm{b}=3 \mu \mathrm{m}$. 
Observaciones. Nuestro material coincide bien con la descripción de Senn-Irlet (1995). Sus características macro y microscópicas difieren en algunos detalles con recolectas previas de este taxon en Canarias, en ecología diferente (bosque de pinar) (Beltrán \& al., 2003).

Ecología. Li, Fo (Laurus novocanariensis). II, III, VII. 8-12-2000 (TFC Mic. 9909); 20-1-2001 (TFC Mic. 10736); 1-5-2001 (TFC Mic. 10798); 29-10-2001 (TFC Mic. 10711); 18-7-2002 (TFC Mic. 10841).

Entoloma gomerense Wölfel \& Noordel., Österr. Z. Pilk. 10: 192. 2001

Píleo de 20-30 mm de diámetro, plano-convexo con el centro generalmente deprimido a subumbilicado, algo higrófano y notoriamente estriado por trans- parencia en todo el radio del píleo, de un azul oscuro, débilmente tomentoso y subescamoso en torno al disco. Láminas distantes, adheridas y a menudo con un pequeño diente decurrente, rosadas con matices azul grisáceos, serruladas y teñidas de azul en la arista. Estípite de 40-50 × 2,5 mm, cilíndrico, más o menos concolor al píleo, glabro. Esporas de 9,2-11,2 × 6,7$7,3 \mu \mathrm{m}$, heterodiamétricas, penta a hexagonales, lisas. Arista de las láminas estéril o heteromorfa. Basidios claviformes, × 9-10 $\mu \mathrm{m}$. Queilocistidios claviformes a anchamente claviformes, subesferopedunculados, raramente cilíndricos, de 18-26 × (6)8-11,5(13) $\mu \mathrm{m}$, notoriamente pigmentados intracelularmente de azul; estos elementos se observan asimismo en las caras de las láminas, de forma dispersa. Fíbulas ausentes.

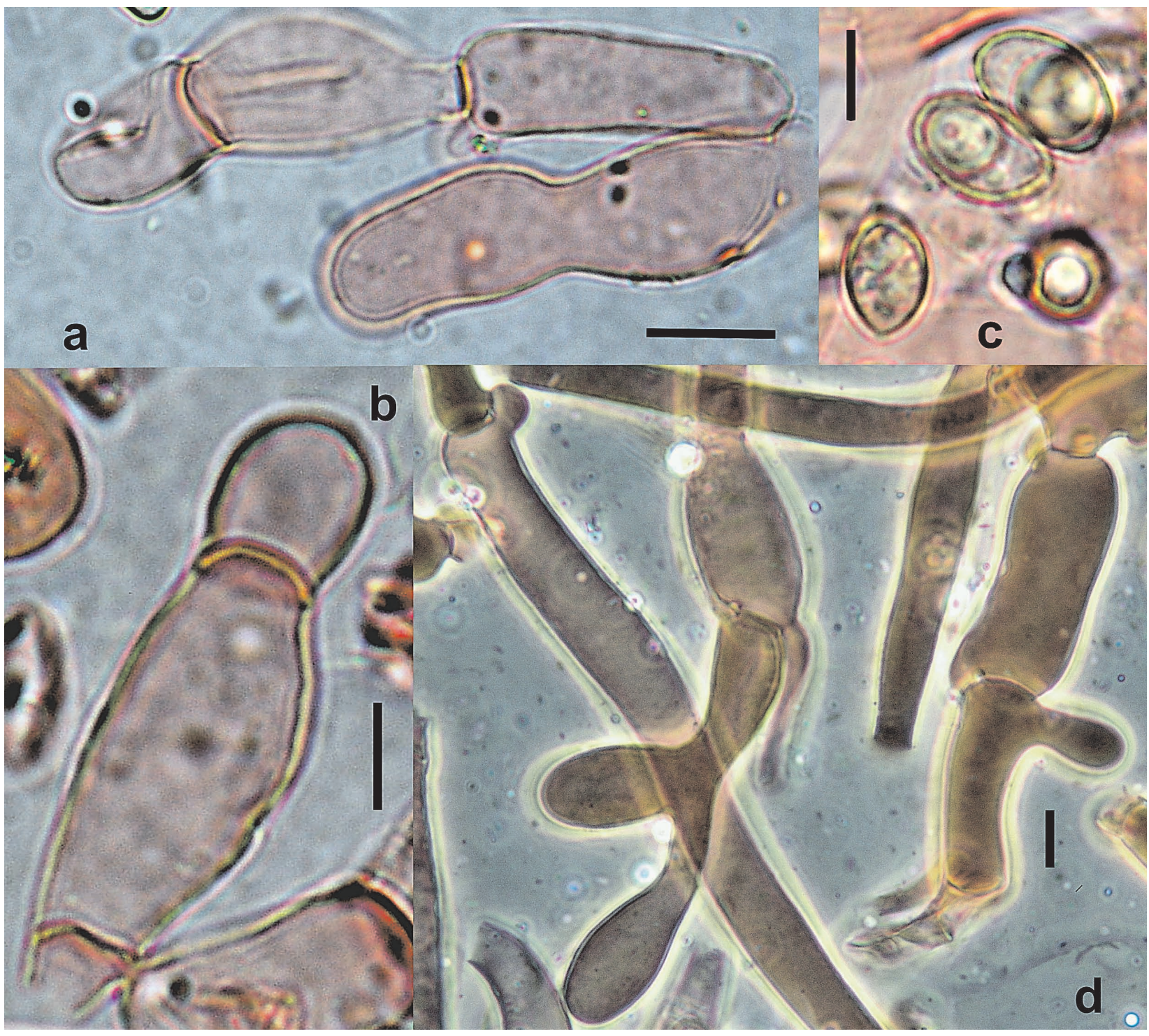

Fig. 9. Crepidotus lundelii: a, queilocistidios; b, queilocistidios; c, esporas; d, pileipellis. Bañares 10798 (TFC Mic.). Barras: a = 10 um; $\mathrm{b}-\mathrm{d}=5 \mu \mathrm{m}$. 
Observaciones. Nuestro material coincide bien con la descripción de Wölfel \& Noordeloos (2001) y Noordeloos (2004) de este taxon del subg. Leptonia (Fr.: Fr.) Noordel., secc. Cyanula (Romagn.) Noordel., stripe serrulatum. Se diferencia de E. serrulata (Pers.) Hesler, presente en la zona de estudio, por su píleo finamente tomentoso y completamente estriado por transparencia, incluso en estadios inmaturos, la arista de las láminas es estéril o heteromorfa y sus queilocistidios más cortos, generalmente claviformes; sorprendentemente, en nuestro material se presentan elementos de morfología exacta a los queilocistidios, en las caras de las láminas, asemejando pleurocistidios. Probablemente, algunas citas previas de E. se- rrulata para Canarias pudieran corresponder al taxon recientemente descrito.

Ecología. Te. III. 12-11-2000 (TFC Mic. 9822).

Entoloma juncinum (Kühner \& Romagn.) Noordel., Persoonia 10: 255. 1979 (Fig. 10)

Píleo de 3-4 cm de diámetro, cónico-convexo a subhemisférico, después extendido y algo deprimido en el centro, marrón grisáceo oscuro, beige a marrón oscuro cuando seco, higrófano, liso y glabro, cuando húmedo estriado por transparencia hasta casi el centro. Láminas sublibres, ventricosas, de 6-8 $\mathrm{mm}$ de ancho, de un marrón-rojizo; lamélulas escasas. Estípite de 50-60 × $4 \mathrm{~mm}$, cilíndrico y algo ensanchado en la

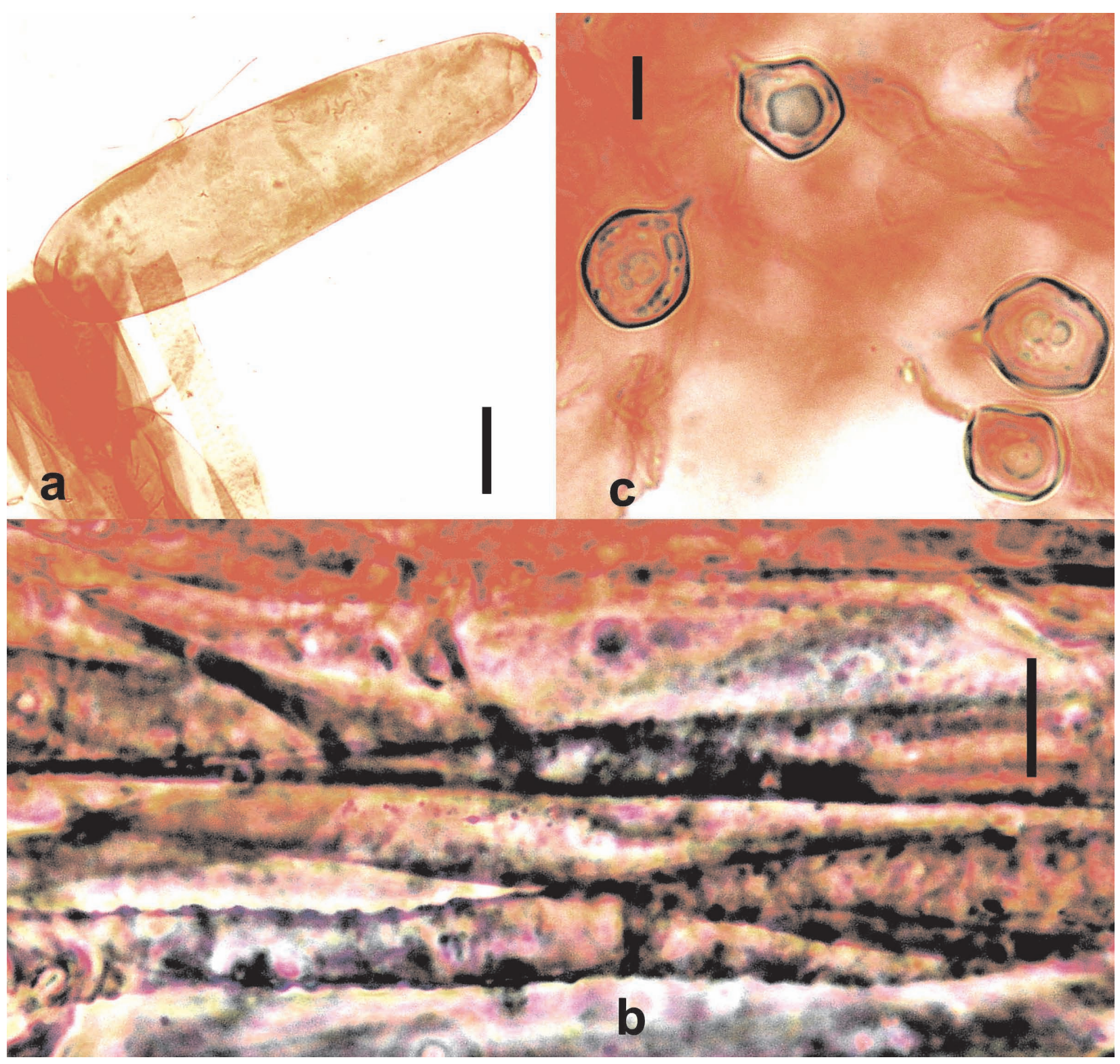

Fig. 10. Entoloma juncinum: a, pileipellis; b, pileipellis; c, esporas. Bañares 10743 (TFC Mic.). Barras: $a=20 \mu \mathrm{m} ; b=10 \mu \mathrm{m} ; \mathrm{c}=5 \mu \mathrm{m}$. 
base, concolor al píleo y provisto de fibrillas blanquecinas dispuestas longitudinalmente y asimismo canaliculado. Esporas de 8,9-11,1 × 7,8-9,6 $\mu \mathrm{m}$, más o menos isodiamétricas, generalmente penta-hexagonales con apícula notoria. Esporada ocre-rosado. Cistidios ausentes. Pileipellis de elementos cilíndricos $(\times 7-18$ $\mu \mathrm{m})$, sin fíbulas, provistos de pigmento incrustante e intracellular; algunos muy gruesos, de hasta $35 \mu \mathrm{m}$, a veces exertos, hialinos, no pigmentados. Basidios de $28-38 \times 10-10,8 \mu \mathrm{m}$, generalmente algo ventricosos, provistos de fíbulas.

Observaciones. Nuestro material coincide bien con la descripción de Noordeloos (1992) y Breitenbach \& Kränzlin (1995).

Ecología. Te. V. 21-1-2001 (TFC Mic. 10743).

Entoloma papillatum (Bres.) Dennis, Bull. Soc. mycol. Fr. 69: 162. 1953

Píleo de 1,5-2 cm de diámetro, campanulado y provisto de una papila prominente más o menos puntiaguda, marrón, higrófano y oscuramente estriado por transparencia cuando húmedo y palideciendo a marrón grisáceo al secarse, liso y brillante. Láminas rosadas, ascendentes, adheridas con diente, ventricosas. Estípite de 35-40 × 1,5-2 mm, concolor al píleo, cilíndrico, liso, pruinoso en el ápice. Esporas de 10,9$12,6(13,6) \times 7,4-8,7(9,2) \mu \mathrm{m}$, hexa-octogonales. Arista fértil. Cistidios ausentes. Basidios de 30-33,3 × $10,8-11,4 \mu \mathrm{m}$, provistos de fíbulas. Pileipellis una cutis de hifas cilíndricas, a veces algo infladas, de 4,3-13 $\mu \mathrm{m}$ de diámetro, las más delgadas provistas de pigmento incrustante muy refringente y también algunas hifas presentan pigmento intracelular, granuloso; fíbulas no observadas en la suprapellis.

Observaciones. Nuestro material coincide bien con la descripción dada por Noordeloos (1992); no obstante, en la suprapileipellis de nuestro material no se observan fíbulas y las hifas presentan pigmento parietal e intracellular.

Ecología: Te. III. 28-11-2000 (TFC Mic. 10781).

Entoloma strigosissimum (Rea) Noordel., Persoonia 10: 211. 1979 (Fig. 11)

Píleo de 1-1,3 cm de diámetro, cónico-campanulado, a veces algo mamelonado, marrón oscuro a marrónrojizo oscuro, enteramente cubierto de pelos más o menos concolores o algo más rojizos; margen incurvado. Láminas gruesas, marrones, ascendentes y adheridas o algo decurrentes con diente. Estípite de 50-60 × 2-2,5 $\mathrm{mm}$, concolor al píleo e igualmente revestido de abundantes pelos. Esporas de 13,3-17,4 × 8,5-10,3 $\mu \mathrm{m}$, marrones, de paredes gruesas y fuertemente nodulosas.
Basidios de 12-15 $\mu \mathrm{m}$ de ancho; basidiolos abundantes. Queilocistidios claviformes, anchamente claviformes, ovoides, obpiriformes, de 14-20(32) $\mu \mathrm{m}$ de ancho, a veces con un largo apéndice apical. Pileipellis de hifas tabicadas, de células cilíndricas 39-111 × 12,2-24,4 $\mu \mathrm{m}$ (elementos terminales cilíndricos o atenuados hacia el ápice), de paredes gruesas, rara vez provistas de pigmento débilmente incrustante, mezcladas con largos pelos setiformes, de hasta $1600 \times 13,3 \mu \mathrm{m}$ (base) $\times 5,5$ $\mu \mathrm{m}$ (ápice), tabicados (células de 55-310 $\mu \mathrm{m}$ ), de paredes gruesas y desprovistas de pigmento incrustante. Estipitepellis provista de pelos setiformes semejantes a los de la pileipellis. Fíbulas ausentes.

Observaciones. Nuestro material coincide bien con la descripción dada por Noordeloos (1992) de este taxon del subgénero Puzarella (Mazzer) Noordel., secc. Dysthales (Mazzer) Noordel. muy característico por presentar todo el carpóforo cubierto de pelos setiformes rígidos. En esta sección se diferencia de especies próximas (E. pseudodysthales Noordel., Tabarés \& Rocabruna y E. dysthaloides Noordel.) básicamente por la pileipellis provista de pelos setiformes de paredes gruesas, no incrustadas.

Ecología. Te. III. 16-5-2001 (TFC Mic. 10727).

Galerina cedretorum var. bispora A.H. Sm. \& Singer, Monogr. Gen. Galerina: 274. 1964 (Fig. 12)

\section{$=$ G. badipes $(F r$.) Kühner, sensu Kühner}

Píleo de 6-9 mm de diámetro, hemisférico a cónicocampanulado, a veces algo umbonado o puntiagudo, de un marrón-rojizo oscuro, de superficie lisa, higrófana y estriada por transparencia, margen incurvado. Láminas espaciadas, anchamente adheridas a subdecurrentes, más o menos concoloras al píleo. Estípite de $12-25 \times 2 \mathrm{~mm}$, cilíndrico, más o menos concolor al píleo, pruinoso en el ápice y algo fibriloso hacia la base, provisto de un anillo membranoso más o menos persistente. Carne insípida. Basidios bispóricos, de $26-34 \times 6-8,6 \mu \mathrm{m}$. Esporas de 8,8-11,4(13) × 5,2-6,3 $(6,6) \mu \mathrm{m}$, amigdaliformes, sublisas a débilmente verrucosas. Queilocistidios lageniformes a fusilageniformes, de ápice flexuoso, cilíndrico o subcapitado, de 41-52 × 8,5-11,2 × 3,4-6,4 $\mu \mathrm{m}$. Pleurocistidios semejantes, generalmente algo más gruesos. Caulocistidios presentes, a veces de ápice mucoso-granuloso. Fíbulas presentes en la pileipellis.

Observaciones. Siguiendo a Gulden (1980) y Bon (1992), la presencia de bucles, pleurocistidios, esporas de placa suprahilar lisa, subrugulosas y estrechas, con poro germinativo indistinto, basidios constantemente bispóricos y su sabor insípido nos lleva a este taxon del subg. Galerina ss. lato, secc. Naucoriopsis 
Kühner. Destaca no obstante en nuestro material la presencia de un anillo más o menos membranoso muy patente en los ejemplares jóvenes, que recuerda a las especies de la estirpe marginata, resultando difícilmente diferenciable de las bispóricas G. acre Gulden, de sabor acre, y $G$. badipes (Fr.) Kühner, non sensu Kühner, de esporas verrucosas. G. unicolor (Vahl) Singer, ampliamente citada para Canarias, se diferencia por sus basidios 2-3-4 espóricos esporas verrucosas de perisporio suelto y queilocistidios fuertemente capitados. Esta especie se encuentra asimismo en la zona de estudio, presentando una de las recolectas de queilocistidios a veces tabicados, de ápice notoriamente capitado $(\times 7-8 \mu \mathrm{m})$, a veces mucoso-granuloso, pleurocistidios semejantes pero muy escasos y basidios con 2-3-4 largos esterigmas, de hasta $7 \mu \mathrm{m}$ (TFC Mic. 9921).
Ecología. Li. IX. 19-1-2001 (TFC Mic. 10723). Nueva cita para la isla de La Gomera.

Galerina sahleri (Quél.) Kühner, Beitr. Kryptfl. Schweiz 10(3): 136. 1948

= G. calyptrospora Kühner; G. bypnorum f. calyptrospora (Kühner) Kühner

Píleo de 3-5 mm, cónico, subpapilado a obtusamente mamelonado, de color anaranjado a ocre-leonado y margen más claro, higrófano y estriado por transparencia. Láminas 7-8 (5-6 lamélulas), ascendentes, adnatas con un pequeño diente, más o menos concoloras al píleo. Estípite de 10-15 × 0,5-0,8 mm, cilíndrico, concolor al píleo o algo más claro, casi hialino y el ápice notoriamente pruinoso. Esporas 9,1-10,7 × 5,9-6,6 $\mu \mathrm{m}$, elipsoides a amigdaliformes, de un ocre

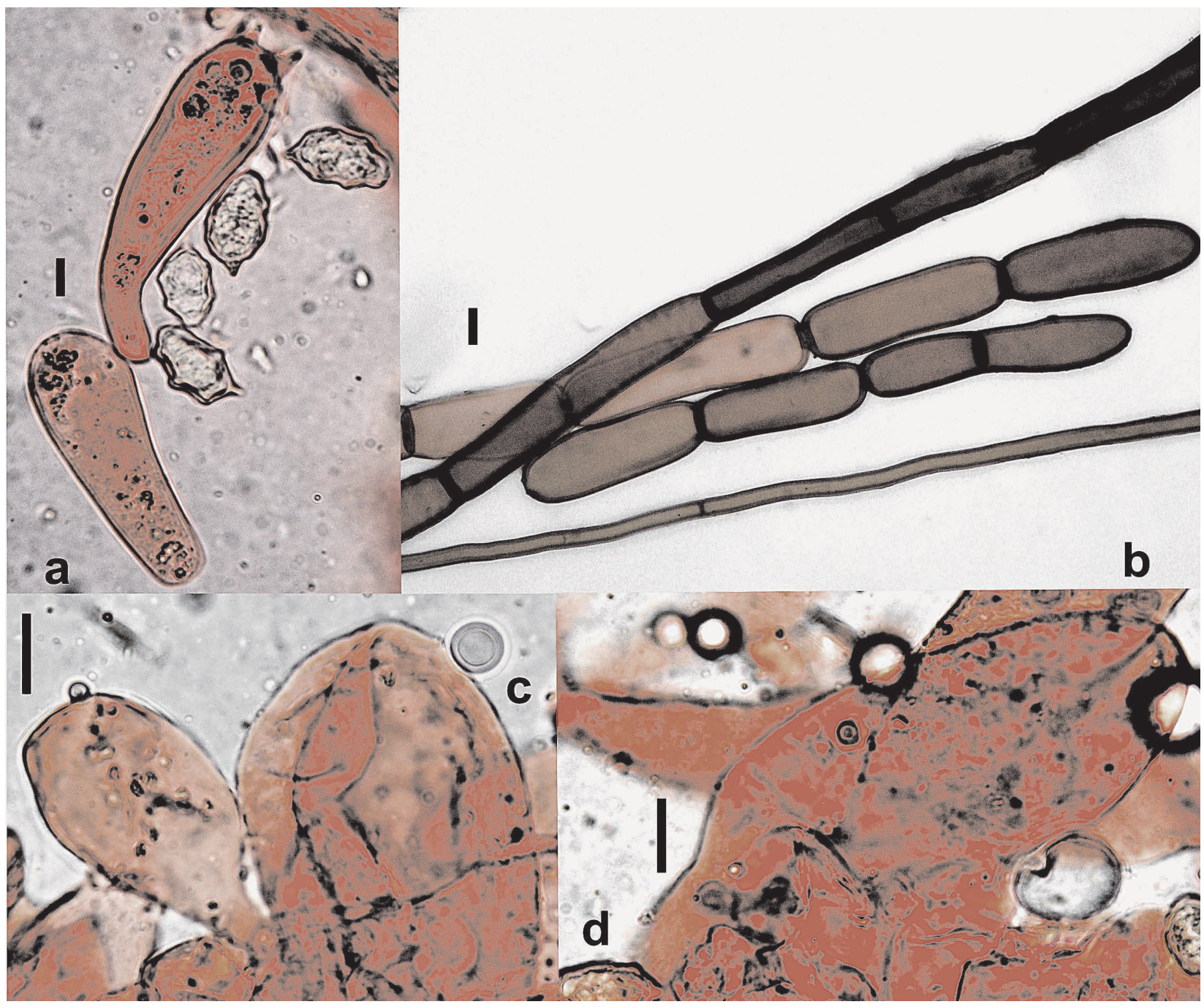

Fig. 11. Entoloma strigosissimum: a, basidio, basidiolo y esporas; b, pileipellis; c, d, queilocistidios. Bañares 10727 (TFC Mic.). Barras: $a=5 \mu \mathrm{m} ; \mathrm{b}-\mathrm{d}=10 \mu \mathrm{m}$. 
claro, lisas a sublisas (débilmente punteadas), ápice a veces obtuso y a menudo caliptradas. Esporada marrón-ocrácea. Queilocistidios lageniformes, capitados (ápice de 5-8 $\mu \mathrm{m}$ de ancho). Pleurocistidios ausentes. Revestimiento pileico de hifas más o menos cilíndricas, de 52-74 × 3,7-11 $\mu \mathrm{m}$, con pigmento parietal y provistas de fíbulas.

Observaciones. Algunos autores (Watling \& Gregory, 1993; De Haan \& Walleyn, 2002) incluyen este taxon en G. bypnorum (Schrank) Kühner. No obstante seguimos a Bon (1992), quien sitúa G. sableri en el subg. Mycenopsis (Smith \& Singer) Bon, secc. Calyptrospora Smith \& Singer. Diferenciamos nuestro material de G. bypnorum, citado para Canarias por Beltrán $\&$ al. (2004), por sus esporas lisas a sublisas, a menudo caliptradas y queilocistidios notoriamente capitados. Citas previas de G. bypnorum para Tenerife (Cool, 1924) y La Gomera, concretamente para la zona de estudio (Bañares \& al., 1980), desprovistas de descripción y testimonio de herbario, pudieran corresponder a este taxon. G. rubiginosa (Pers.) Kühner [G. vittiformis (Fr.) Earle], asimismo citada para Canarias (Baña-

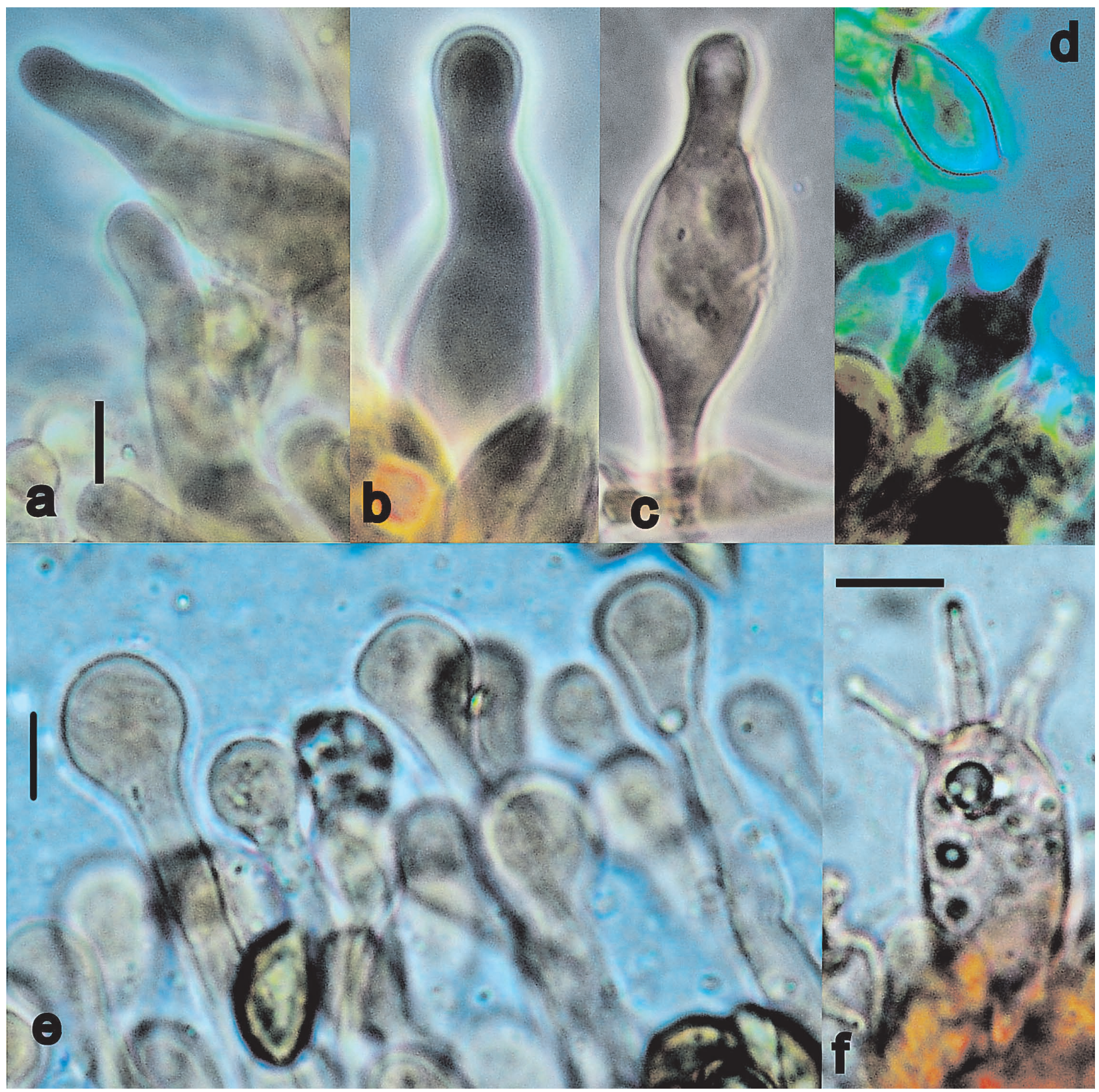

Fig. 12. Galerita cedretorum var. bispora: a, b, queilocistidios; c, pleurocistidio; d, espora y basidio. G. unicolor: e, queilocistidios; f, basidio. [a-d, Bañares 10723 (TFC Mic.); e, f, Bañares 9921 (TFC Mic.)]. Barras: a-f $=5 \mu \mathrm{m}$. 
res \& al., 1986), se diferencia por el estípite enteramente pruinoso, presencia de pleurocistidios y esporas mayores.

Ecología. Mu (Polytrichum sp.). V. 21-1-2001 (TFC Mic. 10725).

\section{Conclusiones}

Considerando el trabajo recientemente publicado por nosotros (Beltrán \& al., 2008) y el catálogo actualizado que aquí presentamos, un total de 45 táxones de Agaricales s.l. se citan por primera vez para la isla de La Gomera, de las que 11 lo son para las Islas Canarias: Clitocybe cistopbila, C. nivea, Galerita sableri, Hygrocybe chlorophana, $H$. reidii, Lepiota pseudolilacea, Marasmius corbariensis, M. scorodonius var. virgultorum, Mycena baematopus, M. mirata y Pluteus insidiosus. Por su lado, el catálogo del Parque Nacional se incrementa en 52 especies. En esta contribución se cita por primera vez para La Gomera Galerina cedretorum var. bispora; asimismo, tras una revisión de material del herbario TFC Mic., se excluyen las citas para esta isla de los táxones Clitocybe rhizophora (Velen.) Joss., ss. Joss., confundido con C. pruinosa; Hygrophorus bypothejus (Fr.) Fr. var. aureus (Arrh.) Imler, posiblemente confundido con $H$. chlorophana; $H$. citrina (Rea) Lange, nomen ambiguum (Boertman, 2000), posiblemente confundido con alguna especie del subg. Hygrocybe, secc. Chlorophaninae (Herink) Arnolds ex Candusso, y finalmente H. conica (Schaeff.: Fr.) Kumm. var. chloroides (Malençon) M. Bon, sinónimo taxonómico de la especie tipo.

Con un total de 139 táxones, los Agaricales s.l. constituyen uno de los grupos mejor representados de las micobiota del Parque Nacional, precedido por los Aphyllophorales s.l. (160 táxones) y seguido por los Myxomycetes s.l. (83) y Ascomycetes (58). En cuanto a su riqueza en relación con las dos grandes formaciones de monteverde, los Agaricales s.l. cobran mayor importancia en las formaciones de Laurisilva (81 táxones) que en Fayal-Brezal s.l. (58), siendo estas proporciones semejantes a las que exhiben los Aphyllophorales s.l. En cuanto a los sustratos, 90 constituyen elementos terrícolas, 44 lignícolas, 4 corticícolas, 9 foliícolas, 2 carbonícolas, 6 muscícolas y 1 coprófilo, prefiriendo por tanto los Agaricales s.l. el emplazamiento sobre suelo, frente a los Aphyllophorales s.l., mayormente lignícolas.

\section{Agradecimientos}

Este trabajo forma parte de un proyecto más amplio, dedicado a la biota no vascular (hongos, líquenes y briófitos) del Parque $\mathrm{Na}-$ cional de Garajonay, el cual fue financiado por el Organismo Autónomo de Parques Nacionales en virtud de un convenio de co- laboración con la Universidad de La Laguna (Ref. 1802069932/ 2000).

\section{Referencias bibliográficas}

Bañares, Á. \& Beltrán, E. 1982. Adiciones a la flora micológica canaria. II. Collectanea Botanica 13(2): 423-439.

Bañares, Á. \& Beltrán, E. 1987. Adiciones a la flora micológica canaria. V. In: Actas del VI Simposio Nacional de Botánica Criptogámica: 201-211. Granada.

Bañares, Á. \& Beltrán, E. 1990. Adiciones a la flora micológica canaria. IV. Gomera (Parque Nacional de Garajonay). In: Homenaje al Profesor Dr. Telesforo Bravo I: 117-124. La Laguna.

Bañares, Á. \& Beltrán, E. 1993. Adiciones a la flora micológica canaria. VIII. Con especial referencia a la isla de La Gomera (Parque Nacional de Garajonay). Boletín de la Sociedad Micológica de Madrid 18: 117-133.

Bañares, Á., Beltrán, E. \& Wildpret, W. 1980. Adiciones micológicas para las islas de Tenerife, Gomera y El Hierro (I. Canarias). Vieraea 8(2): 227-336.

Bañares, Á., Beltrán, E. \& Wildpret, W. 1986. Contribución al estudio micológico de los pinares de Tamadaba (Gran Canaria). III. Agaricales (1. ${ }^{a}$ parte). Vieraea 16: 137-160.

Bañares, Á., Beltrán, E. \& Rodríguez-Armas, J.L. 1991. Adiciones a la flora micológica canaria, VII. Boletín de la Sociedad Micológica de Madrid 15: 13-26.

Bellú, F. 1996. Alcune specie mediterranee di recente identificazione con particulare riguardo al genere Clitocybe. Rivista di Micologia 39(2): 99-114.

Beltrán, E. \& Bañares, Á. 1983. Adiciones a la flora micológica canaria III. Lazaroa 5: 291-296.

Beltrán, E. \& Wildpret, W. 1975. Táxones nuevos en la flora fúngica canaria. Vieraea 5(1-2): 127-166.

Beltrán, E., Rodríguez-Armas, J.L. \& Bañares, Á. 2003. Observaciones sobre la sucesión fúngica en pinares después de un incendio: pinares de Fuencaliente (La Palma, Islas Canarias). Boletín de la Sociedad Micológica de Madrid 27: 101-147.

Beltrán, E., Rodríguez-Armas, J.L., Bañares, Á., Barrera, J. \& Ladó, C. 2004. Hongos. In: Beltrán, E. (ed.), Hongos, líquenes y briófitos del Parque Nacional de la Caldera de Taburiente (La Palma, Islas Canarias): 55-232. Madrid.

Beltrán, E., Rodríguez-Armas, J.L., Bañares, Á. \& Ladó, C. 2008. Hongos. In: Beltrán, E. (ed.), Hongos, líquenes y briófitos del Parque Nacional de Garajonay (La Gomera, Islas Canarias): 41 232. Madrid.

Bon, M. 1992. Cle monographique des especes galero-naucorioides. Documents Mycologiques 84: 1-89.

Bon, M. 1997. Flore Mycologique d'Europe. Les Clitocybes, Omphales et ressemblants. Documents Mycologiques (Mémorie hors série 4): 1-181.

Bravo, T. \& Bravo, J. 1990. Esquema geológico del Parque Nacional de Garajonay. In: Pérez de Paz, P.L. (ed.), Parque Nacional de Garajonay. Patrimonio Mundial: 39-46. Madrid.

Breitenbach, J. \& Kränzlin, F. 1995. Fungi of Switzerland (Vol. 4. Agarics, $2^{\text {nd }}$ part.) Mykologia. Lucerne.

Consiglio, G. 1997. Contributo alla conoscenza dei macromiceti della regione Emilia Romagna. IX. Genere Clitocybe. Rivista di Micologia 40(4): 321-337.

Cool, C. 1924. Contributions a la connaissance de la flore mycologique des Iles Canaries. Bulletin de la Societe Mycologique de France 40(1): 129-244.

De Haan, A. \& Walleyn, R. 2002. Studies in Galerina. Galerina Flandriae (1) (Fungi non delineati). Candusso. Allasio. 
Gulden, G. 1980. Alpine Galerinas (Basidiomycetes, Agaricales) with special reference to their occurrence in South Norway at Finse on Hardangervidda. Norwegian Journal of Botany 27: 219-253.

Höiland, K. 1979. Studies in the genus Inocybe (Fr.) Fr. (Agaricales) of the western Canary Islands. Vieraea 8(1): 13-22.

Jiménez C.C., Tejedor, M.L., Rodríguez, A. \& Fernández, E. 1990. Los suelos del Parque Nacional de Garajonay y su entorno. In: Pérez de Paz, P.L. (ed.), Parque Nacional de Garajonay. Patrimonio Mundial: 47-56. Madrid.

Marzol, M.V., Sánchez, J.L. \& Santana, L. 1990. El clima de Garajonay en el contexto insular. In: Pérez de Paz, P.L. (ed.), Parque Nacional de Garajonay. Patrimonio Mundial: 47-56. Madrid.

Migliozzi, V. \& Cambonii, M. 1999. La micoflora del litorale romano. $1^{a}$ Contributo. Bollettino del Gruppo Micologico G. Bresadola - Nuova Serie 42(1): 15-45.

Noordeloos, M.E. 1992. Entoloma s.l. (Fungi Europaei 5). Candusso. Saronno.

Noordeloos, M.E. 2004. Entoloma s.l., Supplemento (Fungi Europaei $5^{\text {a }}$. Candusso. Alassio.

Pérez de Paz, P.L., Del Arco, M., Acebes, J.R. \& Wildpret, W. 1990. La vegetación cormofítica (vascular) del Parque Nacional de Garajonay. In: Pérez de Paz, P.L. (ed.), Parque Nacional de Garajonay. Patrimonio Mundial: 137-172. Madrid.

Pérez de Paz, P.L., Del Arco, M. \& González, R. 2008. Vegetación del Parque Nacional de Garajonay. In: Beltrán, E. (ed.), Hongos, líquenes y briófitos del Parque Nacional de Garajonay (La Gomera, Islas Canarias): 21-32. Madrid.

Rodríguez-Armas, J.L., Beltrán, E., Bañares, Á. \& González, M.D. 1983. Adiciones a la flora micológica canaria. VI. Documents Mycologiques 72: 65-72.

Santana, L. 1990. La importancia hidrológica de las nieblas en las cumbres del Parque Nacional de Garajonay. In: Pérez de Paz, P.L. (ed.), Parque Nacional de Garajonay. Patrimonio Mundial: 67-71. Madrid.

Senn-Irlet, B. 1995. The genus Crepidotus (Fr.) Staude in Europe. Persoonia 16(1): 1-80.

Tabarés, M. 1996. Clitocybe nivea. In: Bolets de Catalunya: lám. 704. Barcelona.

Watling, R. \& Gregory, N.M. 1993. Cortinariaceae p.p. (British Fungus Flora 7). Royal Botanic Garden. Edinburgh.

Wölfel, G. \& Noordeloos, M.E. 2001. Neue oder bemerkenswerte Entoloma-Arten der Kanarischen Inseln. Österreichische Zeitschrift für Pilzkunde 10: 185-200.

Recibido: 11-XI-2008

Aceptado: 3-II-2009 